\title{
DEVELOPMENT OF A QUALITY INSPECTION SYSTEM USING LASER BASED SCANNER
}

\author{
PARK, H. S. \& TULADHAR, U. M.
}

Abstract: This paper presents a novel method for defect detection on oil-pan surface using point cloud data obtained from triangulation based $3 D$ laser scanner. For the detection of shape deviation in the oil-pan surface we used registration method to align the scanned model and reference model into same coordinate frame. Then, the registered models are compared and the error is calculated. For this purpose, the well-known Iterative Closest Point (ICP) algorithm is adopted. A variant of point-toplane ICP algorithm is implemented to obtain higher accuracy in registration process. For enhancing the corresponding point search speed a $k$-dimensional binary tree ( $k d$-tree) search algorithm is used. For the measurement of flatness of mounting surface in oil pan, mean plane reconstruction method is applied using singular value decomposition (SVD) technic. This, mean plane is used as a reference mean flat surface to measure the degree of flatness of the mounting surface of oil pan.

Key words: Point cloud, Iterative-closest-point, kd-tree, Singular value decomposition
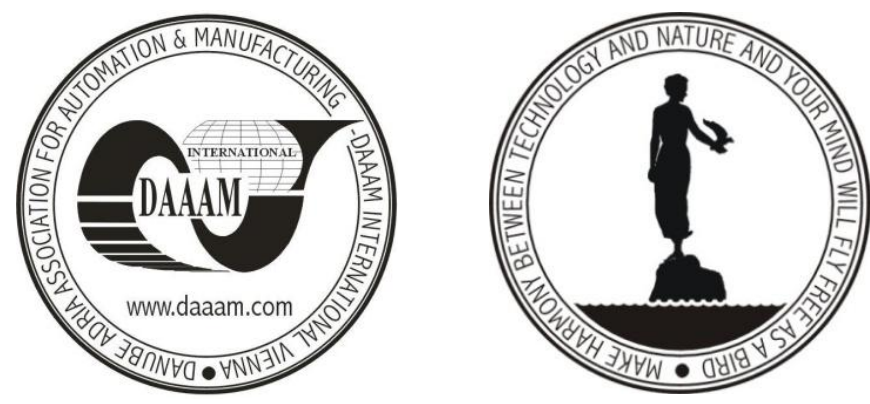

Authors' data: Prof. Dr.-Ing Park, H[ong] S[eok]*; Tuladhar, U[pendra] M[ani]**, *University of Ulsan, Daehak-ro 93, Nam-gu, Ulsan, Republic of Korea, **University of Ulsan, Daehak-ro 93, Nam-gu, Ulsan, Republic of Korea, phosk@ulsan.ac.kr, upendra@mail.ulsan.ac.kr

This Publication has to be referred as: Park, $\mathrm{H}$ [ong-Seok] \& Tuladhar, U[pendra] M[ani] (2014). Development of a Quality Inspection System Using Laser Based Scanner, Chapter 27 in DAAAM International Scientific Book 2014, pp.339-356, B. Katalinic (Ed.), Published by DAAAM International, ISBN 978-3-901509-98-8, ISSN 1726-9687, Vienna, Austria

DOI: $10.2507 /$ daaam.scibook.2014.27 


\section{Introduction}

Laser scanning technologies are becoming important tools in many engineering applications. With the advantage of the amount of data that can be captured within a short time at high rate of accuracy, laser scanners are widely used in many engineering applications such as construction, industries, archaeology, historical studies and many more. (Tomasiak, 2012) discusses a general concept using a 3D scanner in production conditions, as an alternative to inspection and test devices. Various types of laser range finders used in laser scanners are time of flight (ToF), Triangulation and phase range finders. Time of flight scanners use the speed of light and emitted and received beam pulse information. While in the triangulation method the position of laser source and CCD camera is known. Angle made by the laser beam on the object determines the position of the object. Phase scanners compute ranges by measuring the difference in the wave phase between emitted and received beam to find ranges. The laser scanner could be set on a stationary station, or be mounted on a movable device. Depending on the application field, measured ranges vary from centimetres to thousands of meters. The result will be a group of points with particular properties, called point cloud. This point clouds will need a number of processing steps and treatments in which it becomes suitable for using in any specific application (Park \& Tuladhar, 2013).

This chapter presents a study on implementation of triangulation based 3D laser scanner in inspection process of oil pans. For this purpose this research mainly focuses on developing a software system capable of carrying out necessary point cloud processing tasks before an analysis can be done.

Due to the high demand of flawless product, the manufacturing industries have been bound to adopt the better quality inspection system. Consequently, the demand for better automated quality inspection system has risen enormously. With the advancement in the manufacturing technology, now the industries have been able to manufacture products of complex shaped surface which are able to give the dynamic performance. Along with the development of manufacturing system, the necessity of development of inspection system for these complex surfaces has become a challenging job. In last few decades numerous efforts have been made for development of such an automated inspection system. In this research the sample object for the inspection to be carried out is oil-pan. The inspection task consist of shape deviation measurement and the flatness measurement on the surface which is mounted to the main body of the engine.

Currently, a coordinate measuring machine (CMM) and a three-dimensional laser scanner are widely used in the fields of quality inspection system. Touch based CMM systems are highly accurate in comparison to laser based scanning system but they are too slow and can sample very few points. This research was carried out to implement high speed laser measurement system in the inspection process of the oilpan. The laser measuring system yields 3D point cloud data which needs to be preprocessed before inspection task is carried out. The pre-processing method comprises of filtering the un-necessary point cloud data and sampling of the point cloud data. 
Then these processed data were used for inspection with respect to reference models obtained from good parts.

The rest of the chapter is organized as follows. Section 2 gives a literature review of the different techniques used for 3D geometry inspection. Section 3 gives the design procedure of the entire inspection system. In section 4 an improvement of the algorithm discussed in section 3 is given. Section 5 discusses the results and finally the chapter is concluded in section 6 .

\section{Literature review}

In past several years, many researches have been conducted in the field of laser scanner and laser scanned data processing system. A system for gross geometric feature inspection using dense range data was discussed by (Marshall \& Martin, 1991). (Newman \& Jain, 1995) presented an automated visual inspection system for detecting defects of castings by using range images. The system was only applicable to the class of objects containing planar and/or quadric surfaces. Localization was based on the assumption that the expected orientation of the parts was known. Therefore, the system only needed to solve three translational equations and one rotational equation. (Thompson et al., 1999) examined the reverse engineering of designs by generating surface and machining feature information off of range data collected from machined parts. (Langenbach et al., 2001) discussed an automatic visual inspection system for small stampings. This system could detect defects such as cutoffs, incomplete stampings, cracks, and scratches. The localization was a 2-D process. The method of (Osada et al., 2002) creates an abstraction of the 3D model as a probability distribution of samples from a shape function acting on the model. The method introduced in (Prieto et al., 2002) conducted visual inspections of common mechanical parts and made comparisons between measurement surfaces and their CAD models. (Novotni \& Klein, 2004) demonstrated the use of 3D Zernike descriptors. (Kazhdan et al., 2003) compared 3D models with spherical harmonics. (Vucina et al., 2011) used selected common reference points based registration technic to merge multiple point clouds and obtain the complete 3D structure of points cloud which was further processed using triangulation for 3D reconstruction. All the methods only addressed the shape deviation by the matching technique which was not capable of flatness inspection. In this research, our goal is to carryout both the shape and flatness inspection therefore in this research we proposed an automated technique to process the raw point cloud data obtained from the laser scanning system and compare these point cloud data with the existing CAD data for shape deviation whereas for the flatness inspection we have introduced a reference plane construction technique and compare the point cloud of flat surface with respect to this reference plane.

\section{Design and development of the inspection system}

This section consists of a description of the algorithms involved in the development of the inspection system. A sequence of algorithms were implemented 
to realize the entire inspection system. The inspection process comprises of shape error inspection, flatness error inspection and the position error inspection. The shape error is characterized by the deviation of the geometry of the surface of the entire workpiece, whereas the flatness has to be measured in the area where the workpiece has to be mounted with the engine block. Apart from these errors measurement module the software was also developed as per the requirement of processing point cloud data received from the 3D laser scanner. In order to realize the inspection system we need to design and develop each data processing module and then integrated together as a whole system. The schematic diagram shown in Fig. 1. illustrates the necessary processing steps needed to be carried out for the inspection process.

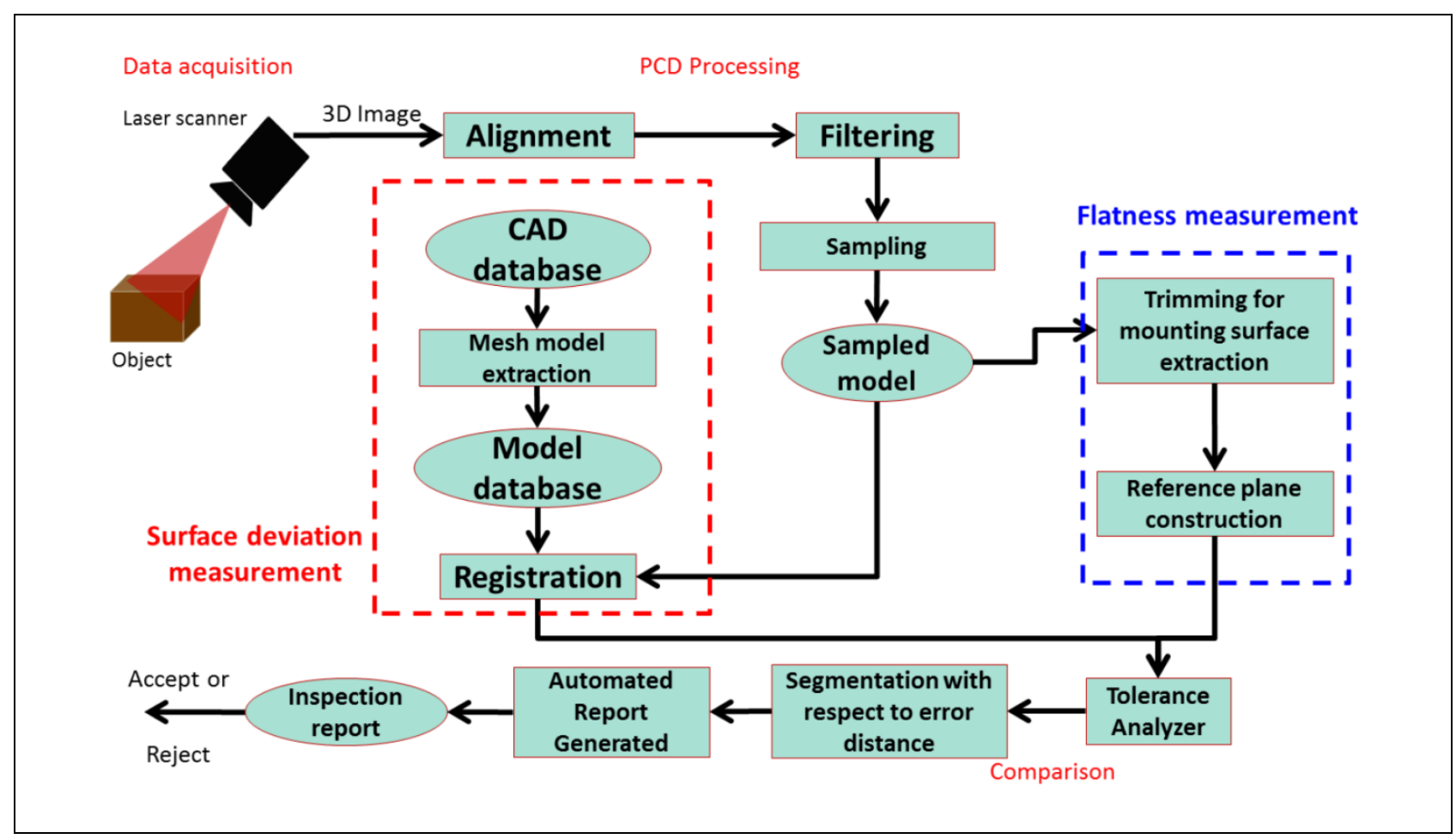

Fig. 1. Schematic diagram to develop inspection system

\subsection{Filtering}

A useful approach to acquire complex geometric models in computer graphics is digitization. From the cloud of points scanned by digitizing devices like laser scanners, a surface description is then obtained by connecting the points in an appropriate manner into a surface mesh, e.g. a mesh of triangles.

Unfortunately, the digitized points often do not reflect the correct location on the real surface, because of physical noise added by the technical scanning device (Vollmer et al., 1999). The effect is that the reconstructed surfaces often do not look satisfactory. The consequence is that the mesh has to be smoothed, with the goal to remove the noise. There are various smoothing algorithms in point cloud processing techniques. In our work we have used the approach of Laplacian algorithm for smoothing the mesh surface obtained after scanning, which is one of the widely used approach for point cloud processing. 
The basic idea of Laplacian based smoothening process in image processing is to replace the grey value of a pixel with an average of the grey values of pixels in some neighborhood. Similarly, the location of a vertex in a finite-element-mesh is corrected by calculating a new location as the average of the locations of vertices in the neighborhood on the mesh. This process of averaging can be applied iteratively, until the result is satisfiable.

The Laplacian algorithm is quite simple and easy to apply: the position $P_{i}$ of vertex $i$ is replaced with the average of the positions of adjacent vertices as shown in Fig. 2. We have

$$
P_{i}= \begin{cases}\frac{1}{|\operatorname{adj}(i)|} \sum_{j \in \operatorname{adj}(i)} q_{j}, & i \in V_{v a r} \\ q_{j}, & i \in V_{v a r}\end{cases}
$$

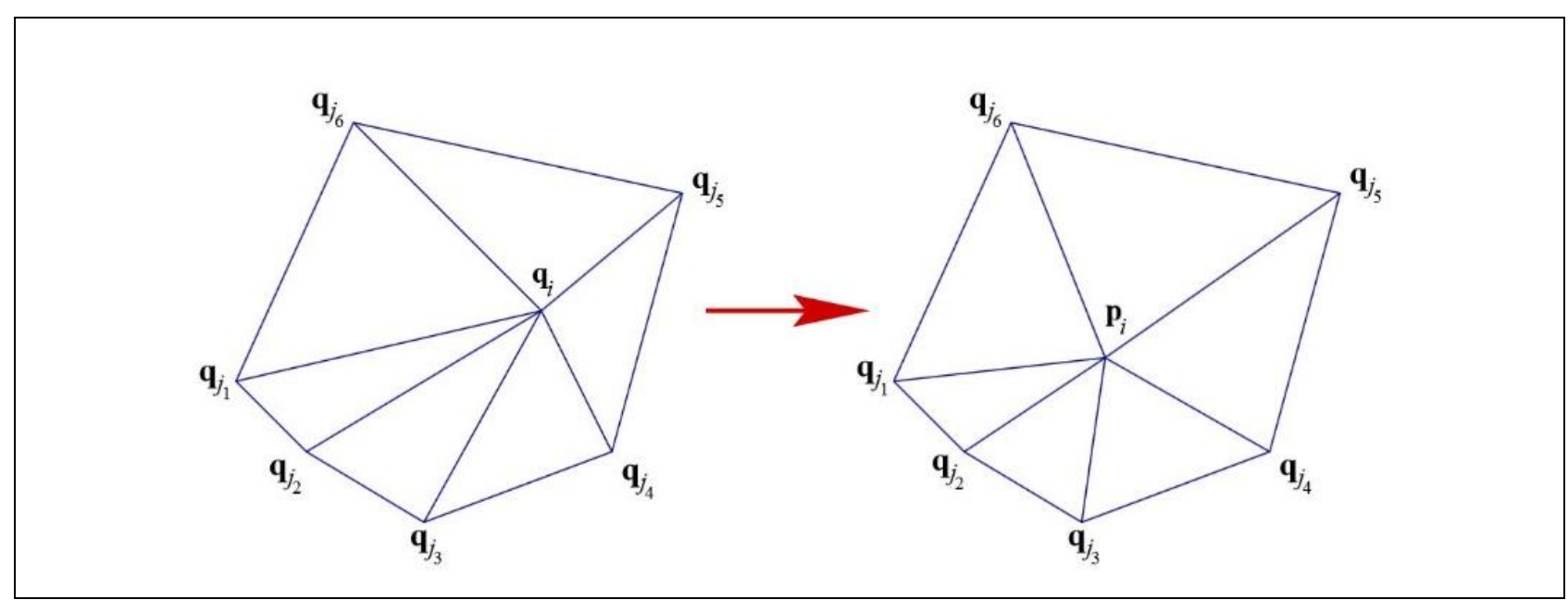

Fig. 2. The laplacian algorithm

There are two techniques to calculate new positions $P_{i}$. The first method is to modify all positions $\mathbf{q} \rightarrow \mathbf{p}$ by one step. So every new position $\mathbf{p}_{\mathbf{i}}, i=1, \ldots, n$, depends completely on the same set of positions, namely q. This method is called the simultaneous version. The second variant is to update the new positions $\mathbf{p}_{\mathbf{i}}$ immediately. This variant is called the sequential version. In this case a position $\mathbf{p}_{\mathbf{i}}$ may not solely depend on the "set" of old positions q but can depend on a previously calculated new position $\mathbf{p}_{\mathbf{j}}$, too. Hence the result of one smoothing pass through all vertices $i \in V_{v a r}$ will depend on the order how the vertices are considered. The simultaneous version needs more storage space for all old positions $\mathbf{q}$ until the new are calculated completely. However, the results of this techniques are better. Surprisingly, among all other smoothing algorithms the Laplacian algorithm solely has the property that the limits of the two techniques are the same if they exist.

Figure 3. (a) shows the original noisy scanned data where the surface contains some noisy points which are deviated from the original surface. These noises are obtained due to the physical noise added by the technical laser devices or if any obstacles are present on the surface during scanning. Figure 3. (b) shows the smoothened surface after the Laplacian filter is applied on the original surface. Here 
10 iterations were carried out to filter the original mesh data. As we can see in this operation some of the screw holes suffers shrinkage. Therefore as the iteration increases the shrinkage of original data is largely increased. Hence, an appropriate number of iterations needed to be chosen for better result.

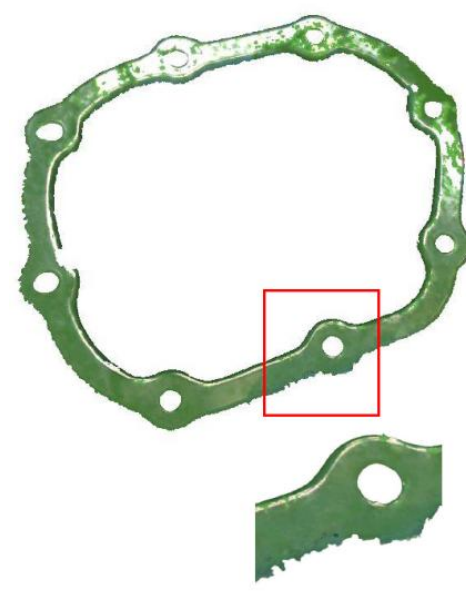

(a)

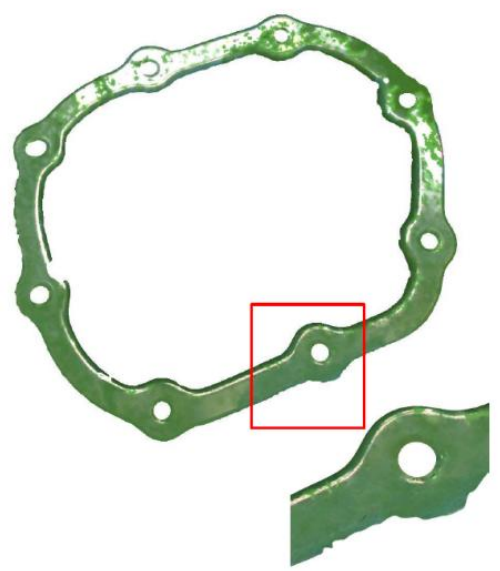

(b)

Fig. 3. The Laplacian filter operation mounting surface (a)Original Mesh (b) Filtered $\operatorname{Mesh}(10$ iterations)

\subsection{Registration}

Measurements on the objects are made relative to the coordinate frame of measurement system (MCS), whereas the nominal CAD model is defined relative to some arbitrary coordinate frame of design coordinate system (DCS) (Park et al., 2013). It is necessary to establish a relationship between these two frames which is known as registration. Registration is the process that involves the alignment of two or more point data sets in different coordinate systems to each other, resulting into one coordinate system for both. In the laser scanning systems, the process is achieved by transforming the coordinate system of one of the points sets to the other point set coordinate system by applying specific translations and rotations (Prieto et al., 2002) in means of rigid body transformations(six degrees of freedom), where the shape and size are preserved(Scale factor is unity - orthogonal transformation).

Thus, in principle, registration is considered as coordinate transformation problem that involves translations and rotations, and the problem is a sub group of the general affine coordinate transformation, solved in the manner of similarity or rigid body transformation (Marshal \& Martin, 1991).

This coordinates transformation problem can be represented as a least square problem which is solved by minimizing the square of error as in Equation 3.

$$
\begin{gathered}
M_{i}=R\left(S_{i}\right)+T \\
\operatorname{Error}(\varepsilon)=\sum_{i=1}^{n}\left\|M_{i}-R\left(S_{i}\right)+T\right\|
\end{gathered}
$$


where, $M$ and $S$ are coordinates of point sets in two different coordinate systems, $R$ and $T$ are rotation matrix and translation vector, respectively.

In the Cartesian coordinate system, rotation in rigid body transformation is normally represented through two ways:

\subsubsection{Euler's angle rotation representation}

Euler angels are rotation angles that applied to each axis to form the desired rotation successively. By applying these angles on the coordinate system rotation axes $X, Y$ and $Z$ successively, the rotation matrix $R$ in Equation 2 is parameterized by:

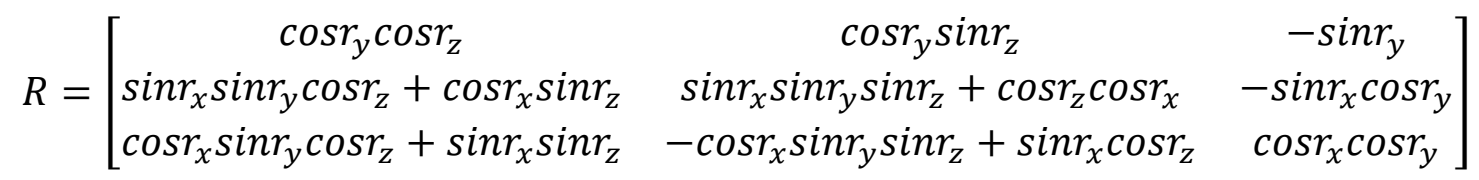

where $r_{x}, r_{y}$ and $r_{z}$ are the rotation angles about $x, y$ and $z$ axes, respectively.

\subsubsection{Axis and angle rotation representation}

Euler angles could suffer from gimbal lock problems when used for rotation representation; an alternative way is to represent the rotation by defining a rotation angle around an arbitrary axis. This can be achieved by using quaternion. (Seokbae et al., 2002)

$$
R=\left[\begin{array}{ccc}
q_{0}^{2}+q_{1}^{2}-q_{2}^{2}-q_{3}^{2} & 2\left(q_{1} q_{2}-q_{0} q_{3}\right) & 2\left(q_{1} q_{3}-q_{0} q_{2}\right) \\
2\left(q_{1} q_{2}+q_{0} q_{3}\right) & q_{0}^{2}+q_{1}^{2}-q_{2}^{2}-q_{3}^{2} & 2\left(q_{2} q_{3}+q_{0} q_{1}\right) \\
2\left(q_{1} q_{3}+q_{0} q_{2}\right) & 2\left(q_{2} q_{3}+q_{0} q_{1}\right) & q_{0}^{2}+q_{1}^{2}-q_{2}^{2}-q_{3}^{2}
\end{array}\right]
$$

and

$$
q_{0}=e_{1} \sin \left(\frac{\theta}{2}\right), q_{1}=e_{2} \sin \left(\frac{\theta}{2}\right), q_{2}=e_{3} \sin \left(\frac{\theta}{2}\right), q_{3}=e_{4} \cos \left(\frac{\theta}{2}\right),
$$

The translation $T$ is parameterized by the translation vector

$$
\left[\begin{array}{lll}
t_{1} & t_{2} & t_{3}
\end{array}\right]
$$

\subsubsection{Iterative closest point(ICP) algorithm for registration}

The iterative corresponding point or better known as the iterative closest point is an effective method used for registration of point clouds. Due to its simplicity it is widely used for the registration of several patches of the point clouds and for the registration of scanned images and their CAD model as well. This algorithm tends to register two different surfaces by finding the closest points of two surfaces and calculating the mean error distance between the surfaces (Besl \& McKay, 1992).

In general, iterative closest point algorithm involves the following steps: 
1. Initialize the cumulative transformation parameters $R$ and $T$ to the identity transformation. Reset the iteration counter, $k$, to zero

2. For each discrete point $S_{i}$ in the Data set, compute the closest point (in terms of Euclidean distance) $M_{i}$ which lies on the surface of the Model.

3 . Using the correspondences from step 2, find a rotation, $R_{k}$, and a translation, $T_{k}$, which minimize Equation 3 via the corresponding point registration method.

2) Find the Closest Points

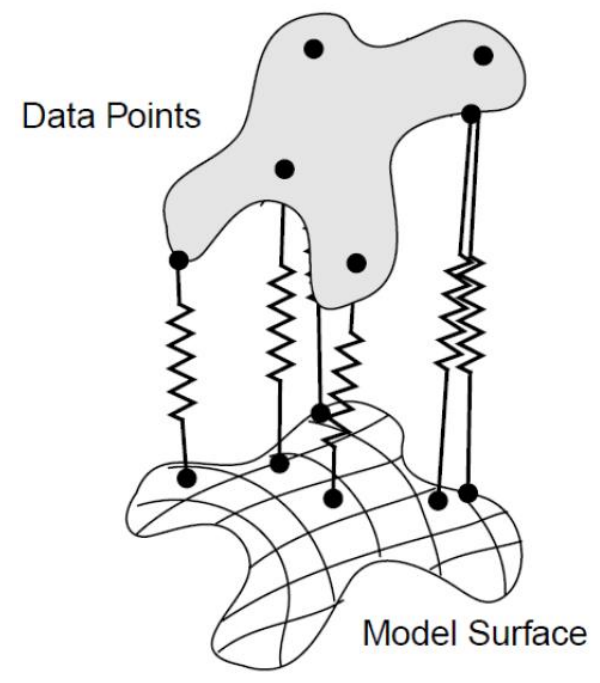

5) IF done THEN exit ELSE GOTO step 2
3 \& 4) Solve the "Corresponding"

Point Problem and Transform Data

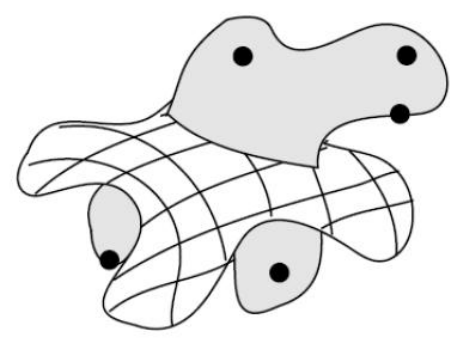

Final Result:

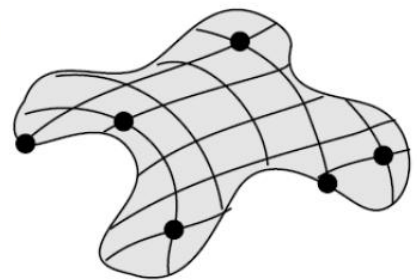

Fig. 4. Demonstration of iterative closest point (ICP) algorithm

4. Apply the incremental transformation from step 3 to all Data points, $S_{i}$ Update the cumulative transformation parameters $R$ and $T$ based upon the incremental transformations, $R_{k}$ and $T_{k}$.

5. If a stopping criterion is satisfied, terminate, else goto step 2 .

Many variants of the iterative closest point are developed since its introduction concerning its robustness and speed. These developments mainly affect the algorithm in one or more of its above mentioned stages. Some developments are on the selection of points used by the algorithm or their matching criteria while others on error minimizing criteria and transformation calculations (Szymon \& Marc, 2001). The iterative closest point can be used with different types of geometrical representations, including points, lines, curves and surfaces. Usually, a combination 
of the target based and feature based methods is used to achieve the best registration results in laser scanning software.

In laser scanning software, the use of iterative closest point is always accompanied with tools to achieve the pre-alignment step. This is done either manually through mouse orientation or by using target points (Kazhdan et al., 2003). Another method for registration in laser scanning is by using modelled features, which are directly modelled or extracted from the point cloud (Yadong \& Piehua, 2006). Depending on the type of the modelled feature, the registration can be carried out either through target registration method or the iterative closest point principle. A method of registration based on the same idea of iterative closest point is to use extracted features as corresponding features. This may overcome the necessity for initial orientation and reduce the searching stage to a small number of features.

\subsection{Shape Deviation Analysis}

Once the object matching has been completed, in order to carry out the shape deviation analysis we need to compute a measure of similarity between two objects based on their shape attributes. The shape matching method proposed here is easy to compute and does not require the solution of the correspondence problem. The proposed distance measure is adapted from the Hausdorff distance between two sets of edge maps (points) associated with the objects of interest. Firstly, a segmentation algorithm is applied to the entire data set to extract the edge points belonging to the object of interest. Then a distance measure algorithm called the modified Hausdorff distance (MHD) (Dubuisson \& Jain, 1994) was applied for matched object analysis. II

While digital image techniques require a one-to-one mapping between the samples of the scanned data and the samples from the CAD version, such a constraint would practically be too restrictive in the case of 3D models. Many methods applied to $3 \mathrm{D}$ models imply a change of topology, in addition to purely geometrical distortions, which justifies the choice of the Hausdorff metric to perform measurements over this type of data, instead of a simple vertex to vertex metric.

\subsubsection{Hausdorff Distance}

Let us first define the distance $d\left(p, S^{\prime}\right)$ between a point $p$ belonging to a surface $S$ and a surface $S^{\prime}$ as:

$$
d\left(p, S^{\prime}\right)=\min _{p \prime \in S^{\prime}}\left\|p-p^{\prime}\right\|_{2}
$$

From this definition, the Hausdorff distance between $S$ and $S^{\prime}$, denoted by $d(S$, $S^{\prime}$ ) is given by:

$$
d\left(S, S^{\prime}\right)=\min _{p \in S}\left(p, S^{\prime}\right)
$$

It is important to note that this distance is in general not symmetrical, i.e. $d(S$, $\left.S^{\prime}\right) \neq d\left(S^{\prime}, S\right)$. We will refer to $d\left(S, S^{\prime}\right)$ as forward distance, and to $d\left(S^{\prime}, S\right)$ as backward 
Park, H. \& Tuladhar, U. M.: Development of a Quality Inspection System Using L...

distance. It is then convenient to introduce the symmetrical Hausdorff distance $d_{s}\left(S, S^{\prime}\right)$, defined as follows:

$$
d\left(S, S^{\prime}\right)=\max \left[d\left(S, S^{\prime}\right), d\left(S^{\prime}, S\right)\right]
$$

The symmetrical distance provides a more accurate measurement of the error between two surfaces, since the computation of a "onesided" error can lead to significantly underestimated distance values, as illustrated by Fig. 5 .

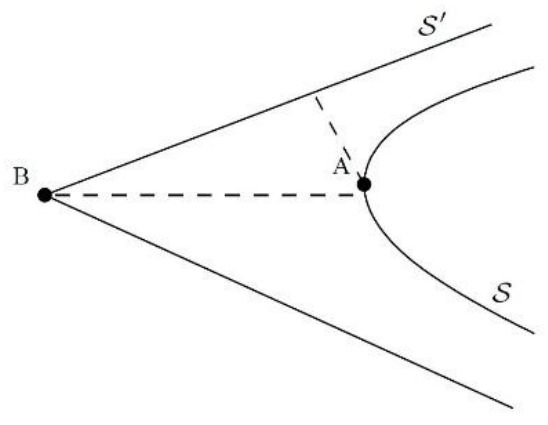

Fig. 5. Demonstration of Hausdroff error distance

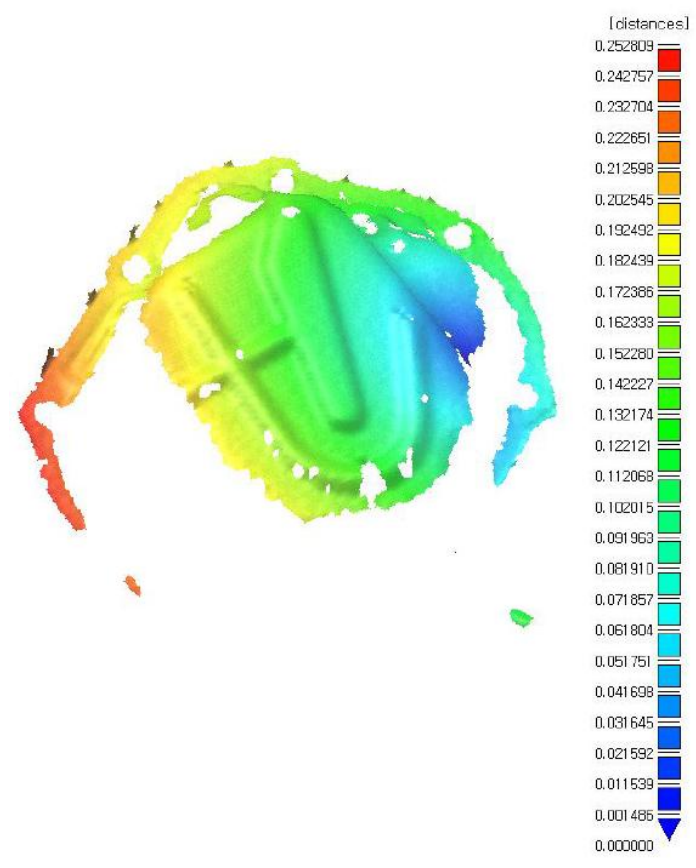

Fig. 6. Shape deviation analysis

In this research we used Hausdorff distance algorithm for the measurement of distance between the scanned and reference model. This distance calculation was used to make an analysis by comparing the scanned data with reference data. Fig. 6 . shows the result after the shape analysis was carried out using Hausdorff distance algorithm. 
The engine oil pan is located at the bottom of the engine and secured by screws. The pan serves as a reservoir for the engine lubricating system and as a housing for the lower internal engine parts. A gasket is mounted between the pan and cylinder block to prevent loss of oil by leaking. To prevent this kind of leaking the mounting surface should be flat enough. Therefore, the manufactured product should go through the flatness check for the quality assurance. Flatness check can be performed with the registration method (Yoon et al., 2013) explained in previous section as well, but registration technique usually yields some error in the alignment of two models therefore it is not possible to obtain précised accuracy in comparison process. Moreover, registration processes use iterative methods to minimize the error distance between scanned and CAD model hence this process costs very high and has comparatively lower accuracy (Shih et al., 2004). Therefore for flatness measurement we proposed to construct a mean flat plane surface that spans along the direction of the mounting surface. The constructed mean plane can be used as a reference flat plane to measure the flatness.

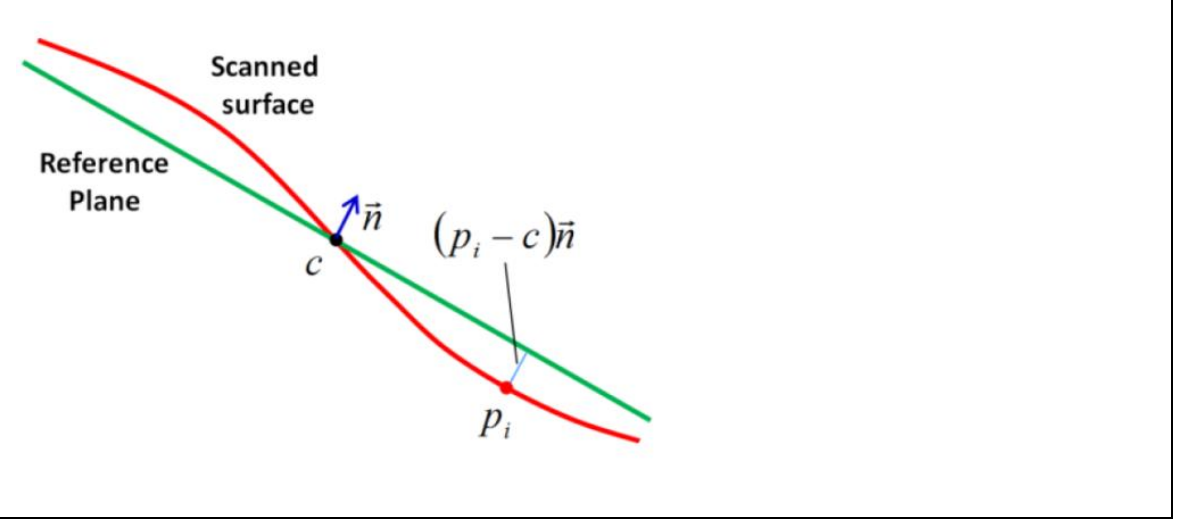

Fig. 7. Representation of reference plane construction using SVD technique

The surface extraction is determined completely by the data points and the choice of planar approximation or interpolation technique (Tang et al., 2009). This paper deals only with the approximation technique. We assume that we want to find a plane (represented by the green line in Fig. 7.) that is as close as possible to a set of $3 \mathrm{D}$ points $p_{1}, p_{2}, \ldots \ldots \ldots, p_{n}$ (represented by the red curve in Fig. 7.) and that the closeness is measured by the squared sum of the orthogonal distances between the points and the plane.

Let the position of the plane be represented by a point $c$ belonging to the plane and let the unit vector $\vec{n}$ be the normal to the plane determining its direction. Then the orthogonal distance between a point $p_{i}$ and the plane is $\left(p_{i}-c\right)^{T} \vec{n}$. Thus the plane can be found by solving

$$
\min _{c,\|n\|=1} \sum_{i=1}^{n}\left(\left(p_{i}-c\right)^{T} \vec{n}\right)^{2}
$$

Solving this for $c$ gives $c=1 / n \sum_{i=1}^{n} p_{i}$.Introducing a $3 \times n$ matrix 


$$
A=\left[\begin{array}{cccc}
p_{1 x}-c & p_{2 x}-c & \ldots \ldots & p_{n x}-c \\
p_{1 y}-c & p_{2 y}-c & \ldots \ldots & p_{n y}-c \\
p_{1 z}-c & p_{2 z}-c & \ldots \ldots & p_{n z}-c
\end{array}\right]
$$

Equation (11) can be formulated as

$$
\min _{\|\vec{n}\|=1}\left\|A^{T} \vec{n}\right\|_{2}^{2}
$$

Using singular decomposition of $A$, we have

$$
A=U S V^{T}
$$

where

$U: m \times m$ orthogonal matrix

$S: m \times n$ diagonal matrix whose diagonal elements are $\sigma_{1}, \sigma_{2}, \sigma_{3}$; in this case,

$$
S=\left[\begin{array}{llll}
\sigma_{1} & 0 & 0 & \ldots . . . \\
0 & \sigma_{2} & 0 & \ldots . . . \\
0 & 0 & \sigma_{3} & \ldots . . .
\end{array}\right]
$$

$V: n \times n$ orthogonal matrix

Hence,

$$
\begin{gathered}
\left\|A^{T} \vec{n}\right\|_{2}^{2}=\left\|V S^{T} U^{T} \vec{n}\right\|_{2}^{2}=\left\|S^{T} U^{T} \vec{n}\right\|_{2}^{2} \\
=\left(\sigma_{1} y_{1}\right)^{2}+\left(\sigma_{2} y_{2}\right)^{2}+\left(\sigma_{3} y_{3}\right)^{2}
\end{gathered}
$$

Where, $y$ is the unit vector $y=U^{T} \vec{n}$. Thus, $\left\|A^{T} \vec{n}\right\|_{2}^{2}$ is minimized for $y=(0,0,1)^{T}$, or equivalently, for $\vec{n}=U(:, 3)$. The minimal value of $\sum_{i=1}^{n}\left(\left(p_{i}-c\right)^{T} \vec{n}\right)^{2}$ is $\sigma_{3}^{2}$.

Since the normal is given by $U(:, 3)$, it follows from the orthogonality of $U$ that the plane is spanned by the two first columns of $U$. 


\subsection{Flatness Analysis}

Fig. 8.(a) shows a point cloud representing the surface of a warped plane. Using the SVD technique discussed above, the mean position ' $c$ ' is calculated from the positions of each point of the cloud. Then the normal orientation of each point is used to calculate a mean normal orientation, which is used to determine the direction of the reference plane.

Fig. 8.(b) shows the result of extracting the reference plane using the point cloud representing a warped surface. Since the mounting surface of an oil pan must be on a flat plane within a given tolerance, therefore this reference plane can be used to detect the flatness error on the mounting surface by calculating the orthogonal distance from each point on the mounting surface to the reference plane.

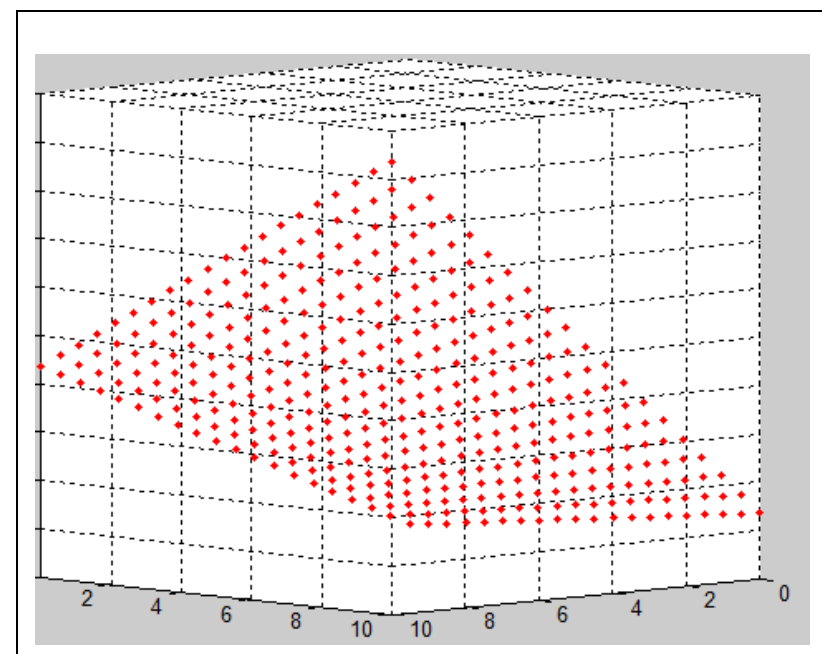

(a)

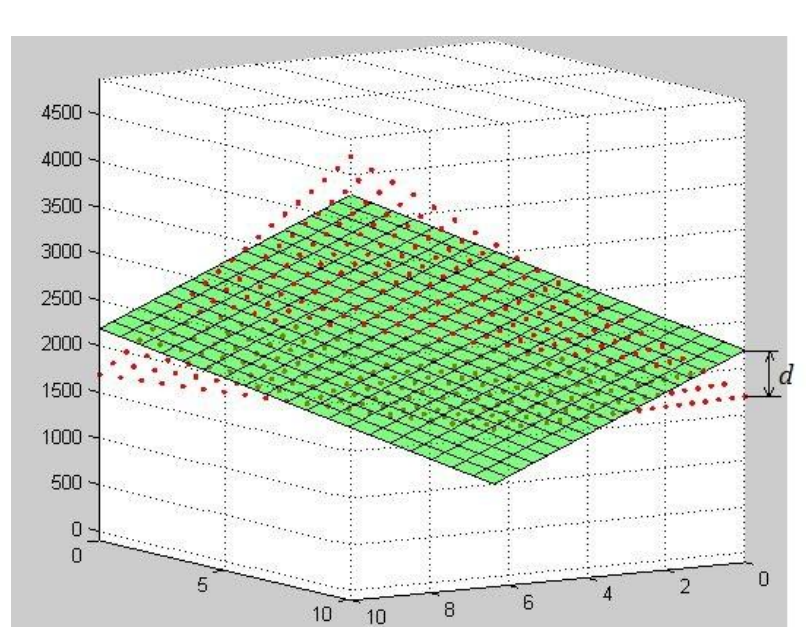

(b)

Fig. 8. Flatness inspection with reference plane construction

\section{Improvement of ICP Algorithm}

Iterative closest point(ICP) algorithm uses a point-to-point distance to calculate the rotational and translational function to find the best pose of a data point with reference to model data. During the process of searching for the best corresponding points in model data, it is possible that the algorithm finds wrong corresponding points (Chen \& Medioni, 1992). An ICP algorithm has many limitations for application. Below given are some criteria for the expected result of this algorithm.

i. If correct correspondences are known, the algorithm can find correct relative rotation/translation.

ii. If two data are initially placed near to each other, this algorithm can converge to give fine registration.

iii. If the two data are not close enough as discussed in case 2 and if the corresponding points are not known as in case 1, ICP algorithm starts the iteration with an assumption that the closest points correspond.

\subsection{Modification of ICP algorithm for enhancing accuracy}

The problem of surface registration consists in finding the transformation which best superposes a surface $S$ with a reference surface $M$. In this work, surfaces used to 
perform registration are represented by point sets or triangle meshes. We call these sets $S=\left\{\mathrm{s}_{\mathrm{i}}\right\}, i=1 \ldots N_{\mathrm{S}}$ and $M=\left\{m_{i}\right\}, i=1 \ldots N_{M}$. A very fruitful approach to tackle this problem is to introduce point correspondences as intermediate variables. Following this idea, Besl and McKay have proposed the ICP algorithm, whose criterion to minimize has been modified in for solving our problem regarding accuracy of the registration algorithm.

As we have discussed in section 3.2, the original ICP algorithm proposed by Besl and McKay, uses the error function of $\operatorname{Error}(\varepsilon)=\sum_{i=1}^{n}\left\|M_{i}-R\left(S_{i}\right)+T\right\|$ where it uses the Euclidean distance between surface points $\left\|M_{i}-R\left(S_{i}\right)+T\right\|$. In this work instead of using the Euclidean distance between surface points, the error distance between normal orientations of the surface at that points is also considered.

In the original ICP algorithm the mean squared error (MSE) objective function is given by:

$$
f(R, T)=\frac{1}{N_{S}} \sum_{i=1}^{N_{S}}\left\|m_{i}-\operatorname{Rot}\left(s_{i}\right)-\operatorname{Trans}\right\|^{2}
$$

Unlike the original ICP algorithm the mean squared error used in this work was Euclidean distance between surface points followed by the Euclidean distance of normal at those points. Below given function is the mean squared error that was used to converge the ICP algorithm in this work.

$$
f(R, T)=\frac{1}{N_{S}} \sum_{i=1}^{N_{S}}\left(\left\|m_{i}-\operatorname{Rot}\left(s_{i}\right)-\operatorname{Trans}\right\|^{2}+\lambda\left\|n_{m i}-\operatorname{Rot}\left(n_{s i}\right)-\operatorname{Trans}\right\|^{2}\right)
$$

Where,

$\left\|n_{m i}-\operatorname{Rot}\left(n_{s i}\right)-\operatorname{Trans}\right\|:$ - Euclidean distance of normal at point $m_{i}$ on surface $M$ and at point $s_{i}$ on surface $S$

$\lambda$ :- Positive value weighing the influence of the normal-to-normal distance term

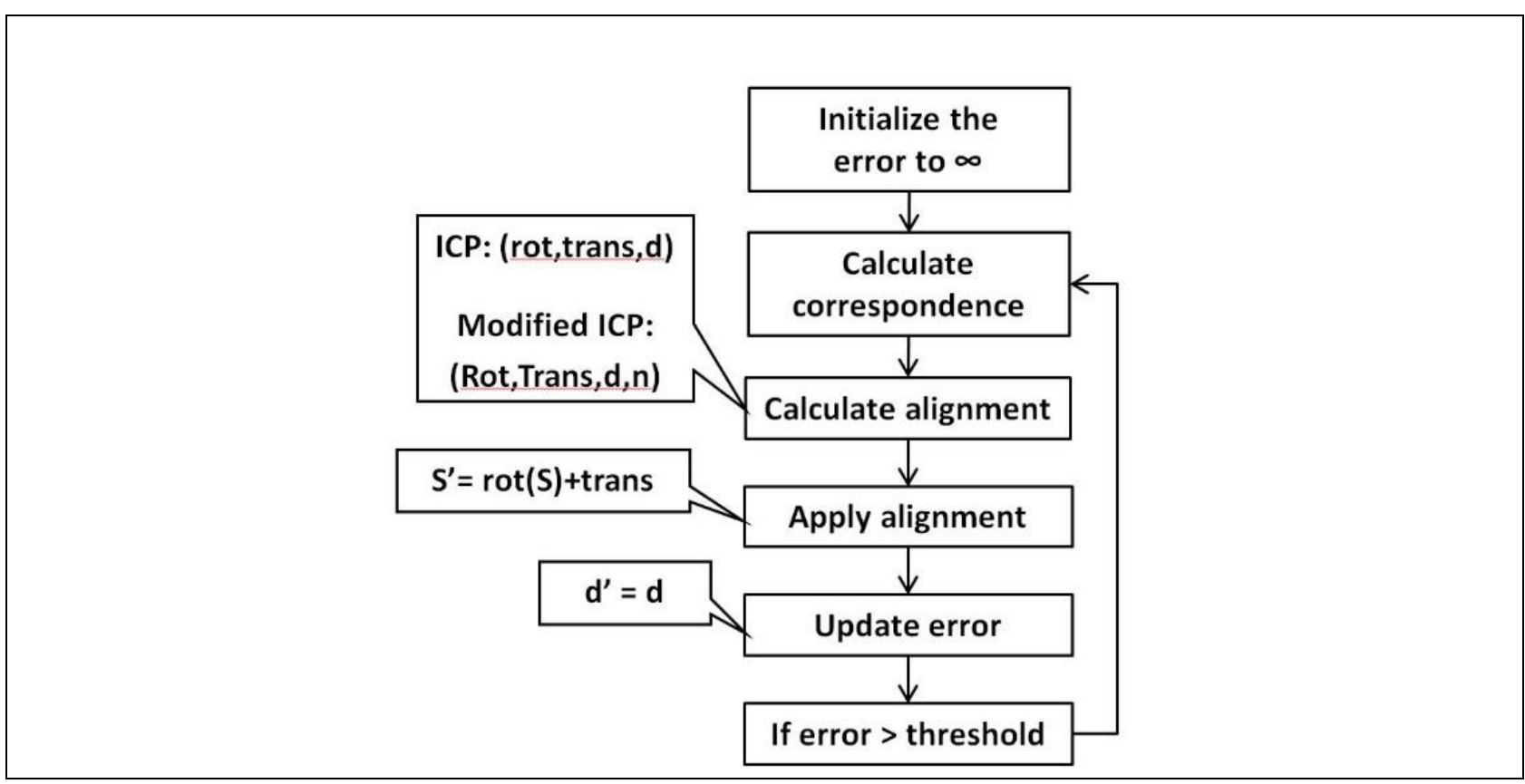

Fig. 9. Schematic diagram to show working procedure of ICP algorithm 


\section{Results and Discussion}

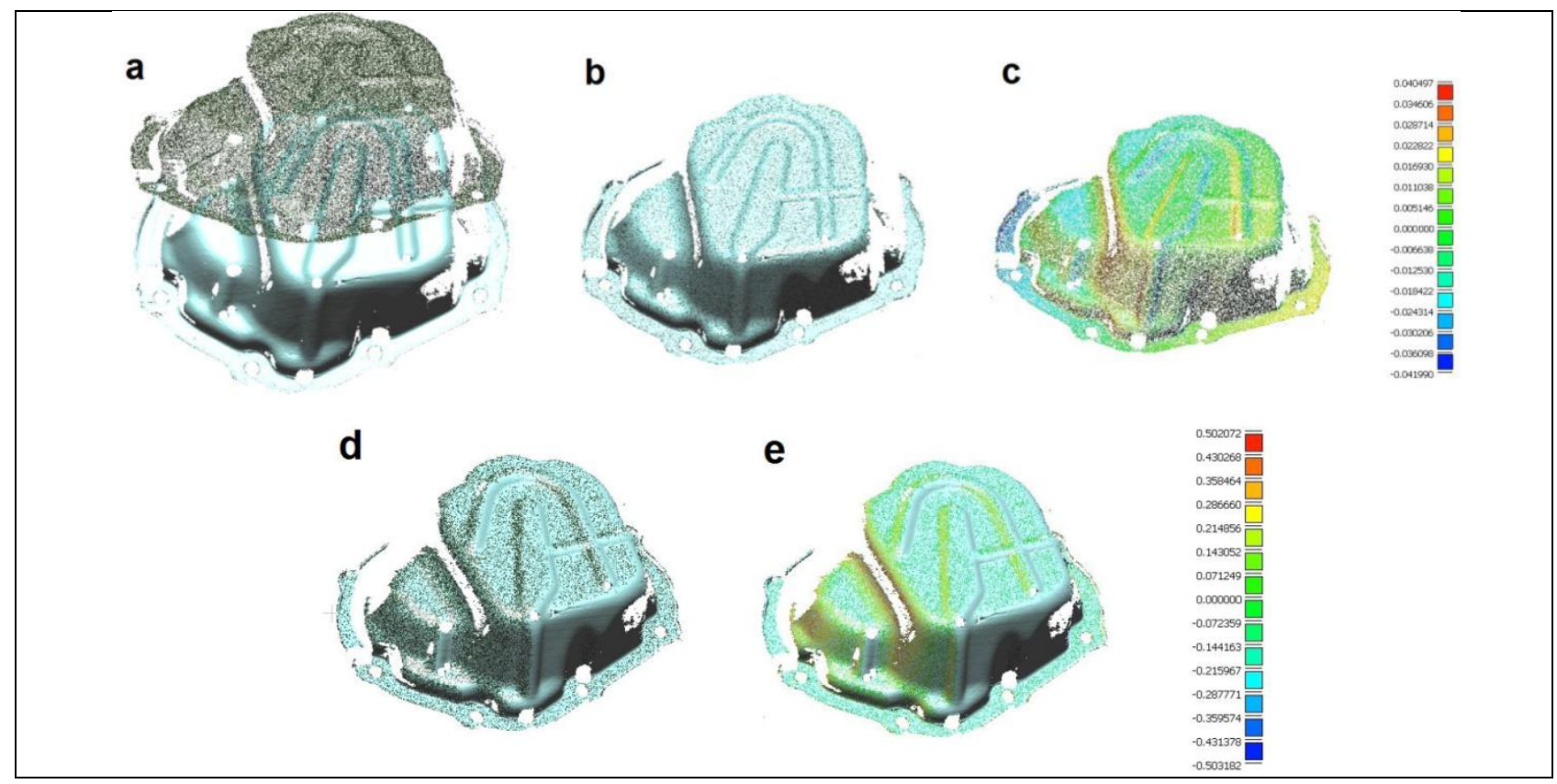

Fig. 10. (a) initial position of two point cloud data (b) position after registration (c) error distance between registered data (d) Registration with original ICP algorithm (Besl and McKay) (e) error result from original ICP

In this section the results of shape deviation analysis and flatness analysis are shown respectively. For the shape deviation analysis the experimental results obtained from the modified ICP algorithm is shown and then the obtained results are compared with the registration result obtained from the point to point ICP algorithm proposed by (Besl \& Mckay, 1992). Fig. 10. shows the comparison between two methods. The comparison shows that it was possible to automatically register both the surfaces very precisely with modified ICP algorithm. In fig. 10., (a) shows the initial position of the two patches of scanned data, (b) shows the registered patches using the proposed modified ICP algorithm, (c) shows the error on the patches during the registration process, (d) shows the registration of patches using point to point ICP method and (e) shows the error result of the point to point ICP method. As we can see in the figure below that the surface interpenetration measure in the proposed method (fig. 10. (b)) is more even in comparison to the Besl and Mckay method in fig. 10. (d). Moreover error distribution value in fig. 10(c) and 10(e), we can see that the maximum error in (c) is $0.041990 \mathrm{~mm}$ whereas in (e) the maximum error value is $0.503182 \mathrm{~mm}$. Besl and McKay method gives higher accuracy when the surfaces to be registered are close enough, whereas our method involves normal orientation of points on each surface better registration result can be applied with this method in comparison to point to point method.

The results obtained from the technique used for flatness measurement in this research were compared with the experimental data obtained from the Coordinate Measurement Machine (CMM) which is currently being used in the company. CMMs are very well known for their high accuracy and are widely used for flatness 
Park, H. \& Tuladhar, U. M.: Development of a Quality Inspection System Using L... inspection. The measurements were carried out in both the good and scrap part and the results are given in the table below.

\begin{tabular}{|c|c|c|c|c|c|}
\hline \multirow{2}{*}{\multicolumn{2}{|c|}{ Measurement type }} & \multirow{3}{*}{$\begin{array}{c}\text { Tolerance } \\
06 \mathrm{~mm}\end{array}$} & \multicolumn{2}{|c|}{ Measurement tool } & \multirow{3}{*}{$\begin{array}{c}\text { Difference } \\
0.0446 \\
\end{array}$} \\
\hline & & & \multirow{2}{*}{$\begin{array}{l}\text { CMM } \\
0.5054 \\
\end{array}$} & \multirow{2}{*}{$\begin{array}{c}\begin{array}{c}\text { Developed } \\
\text { System }\end{array} \\
0.550 \\
\end{array}$} & \\
\hline \multirow{2}{*}{$\begin{array}{c}\text { Flatness } \\
\text { measurement }\end{array}$} & Good Part & & & & \\
\hline & Bad Part & & 0.6532 & 0.730 & 0.0768 \\
\hline
\end{tabular}

Tab. 1. Flatness inspection comparison

The developed system was capable of inspecting the flatness of the samples with acceptable level of accuracy after carrying out several experiments. Fig. 11 shows the graphical image of flatness analysis carried out in the good part (a) and bad part (b) using the developed system.

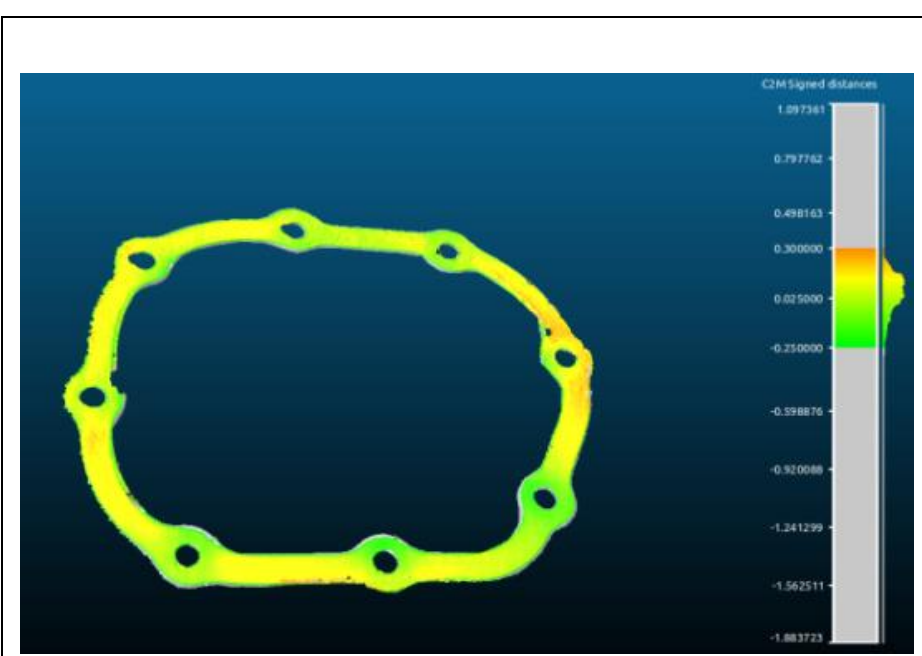

(a)

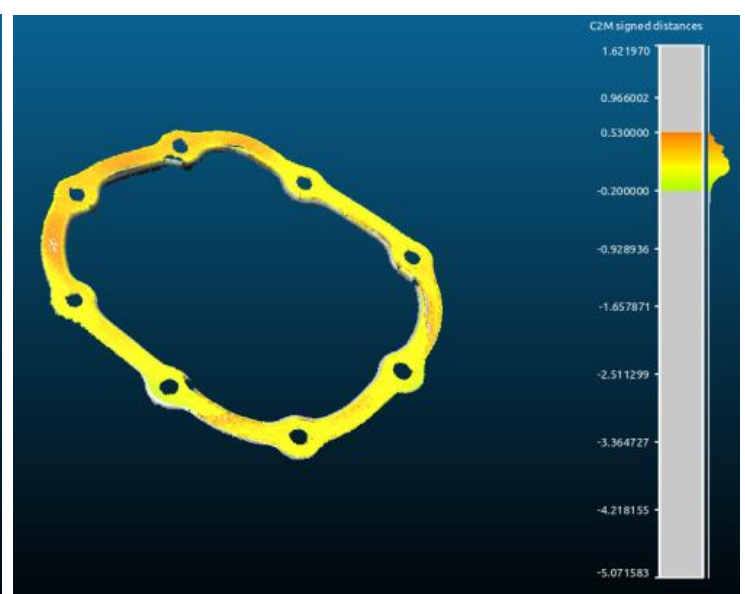

(b)

Fig. 11. Flatness inspection result in (a) good part and (b) bad part

\section{Conclusion}

This project aimed to develop an inspection system which is capable of carrying out inspection task at very high speed while promising the inspection quality as demonstrated by existing CMM system. CMM system is well known for its accuracy in the inspection field. However, the existing CMM system is capable of carrying out only flatness error inspection. Moreover, current system is too old fashioned due to its slow rate of inspection. Therefore, an alternate solution was required to accomplish various inspection task with same system and at greater speed. To address these problems, we introduced high speed laser scanning system for acquiring data from the sample parts and developed an application which is capable of processing the laser scanned 3D data and carryout the inspection in a reliable way.

To carryout both the inspection task within the same system we developed a reliable procedure to use various algorithms and integrated them. The application was 
developed in a conventional way, which follows the traditional point cloud processing techniques however we managed to obtain higher accuracy by optimizing the well-known registration technique of Iterative Closest Point algorithm. In addition, the developed system can bring a great benefit in the field of quality inspection not only for the specific work piece used in this research but to many kind of automotive parts, which need close inspection of geometry and flatness.

Though the flatness measurement technique introduced in this research managed to distinguish "good" and "scrap" part but however still lacks the accuracy level in comparison to the CMM tool. The future task of this research will be to improve the algorithm by introducing the methods to optimize the construction of reference plane for evaluating the flatness of the surface. This research also opens a possibility to further increase the accuracy of the system by introducing various other point cloud pre-processing techniques to obtain optimum 3D shape from the scanned data.

\section{Acknowledgements}

This work (Grant No. 2014-0727) was supported by Business for AcademicIndustrial Cooperative establishments funded Korea Small and Medium Business Administration in 2014.

\section{References}

Besl, P.J. \& McKay, N.D. (1992). A method for registration of 3-D shapes, IEEE Transaction on Pattern Analysis and Machine Intelligence, 14(2), pp. 239-256, Feb 1992

Chen, Y. \& Medioni, G. (1992). Object modeling by registration of multiple range images, Image and Vision Computing, 10(3), pp. 145-155, 1992

Dubuisson, M. P. \& Jain, A. K. (1994). A Modified Hausdorff Distance for Object Matching, Proceeding of International Conference on Pattern Recognition, pp. 566568, 1994

Kazhdan, M.; Funkhouser, T. \& Rusinkiewicz, S. (2003). Rotation invariant spherical harmonic representation of $3 \mathrm{~d}$ shape descriptors, Symposium on Geometry Processing, pp. 156-164 2003

Langenbach, R.; Ohl, A.; Scharf, P. \& Semmler, J. (2001). Automatic visual inspection system for small stampings with free-form surfaces, Proceeding of SPIE 4301, pp. 9-19 2001

Marshall, A. D. \& Martin, R. R. (1991). Automatic inspection of mechanical parts using geometric models and laser range finder data, Image and Vision Computing, 9(6), pp. 385-405, Dec 1991

Newman, T. S. \& Jain, A. K. (1995). A survey of automated visual inspection, Computer Vision Image Underst, 61, pp. 231-62, 1995

Novotni, M. \& Klein, R. (2004). Shape retrieval using 3D zemike descriptors, Computer Aided Design, 36, pp. 1047-1062, 2004 
Osada, R.; Funkhouser, T.; Chazelle, B. \& Dobkin, D. (2002). Shape distributions, ACM Transaction on graphics, 21, pp. 807-832, 2002

Park, H. S.; Tuladhar, U. M. \& Shin, S. C. (2013). Development of An Inspection Method for Defect Detection on the Surface of Automotive Parts, Journal of the Korean Society of Manufacturing Technology Engineers, 22(3), pp. 452-458, May 2013

Park, H. S. \& Tuladhar, U. M. (2013). Development of an Inspection System for Defect Detection in Pressed Parts using Laser Scanned Data, Proc. 24th DAAAM International Symposium on Intelligent Manufacturing and Automation, Katalinic, B. (Ed.), 69, pp. 931-936, Oct 2013

Prieto, F.; Redarce, T.; Lepage, R. \& Boulanger, P. (2002). An automated inspection System, International Journal of Advanced Manufacturing Technology, 19(12), pp. 917-925, June 2002

Seokbae S.; Hyunpung, P. \& Kwan H. L. (2002). Automated laser scanning system for reverse engineering and inspection, International journal of Machine Tools and Manufacture, 42(8), pp 889-897, June 2002

Shih, N. J.; Wu, M. C. \& Kunz, J. (2004). The inspections of as-build construction records by 3D point clouds, Center for Integrated Facility Engineering, Stanford University, Aug 2004

Szymon R. \& Marc L. (2001). Efficient Variants of the ICP Algorithm, Third International Conference on 3D Digital Imaging and Modeling, IEEE, pp. 145-152, 2001

Tang, P. et al. (2009). Characterization of three algorithms for detecting surface flatness defects from dense point clouds, Proc. SPIE7239, Three-Dimensional Imaging Metrology 7239, 72390N-72390N-12, Jan 2009

Thompson, W. B.; Owen, J.; Stark, S.; Henderson, T. \& Germain, H. (1999). Feature based reverse engineering of mechanical parts, IEEE transaction on Robotics and Automation, 12 pp. 57-66, 1999

Tomasiak, J. (2012). Rapid technology in industrial fitting, Proceeding of the 23rd International DAAAM Symposium, Katalinic, B. (Ed.), 23(1), pp 433-436, 2012

Vollmer, J.; Mencl, R. \& Muller, H. (1999). Improved Laplacian Smoothing of Noisy Surface Meshes, Proceedings of Eurographics, 2(18), pp. 131-138, 1999

Vucina, D.; Pehnec, I. \& Gazibaric, D. (2011). Reverse engineering of musical instrument by combining stereo Imaging, $3 \mathrm{~d}$ reconstruction, cad primitives and parametrization, Proceeding of the 22nd International DAAAM Symposium, 22(1), pp 1045-1046, 2011

Yadong L. \& Piehua G. (2006). Automatic Localization and Comparison for FreeForm Surface Inspection, Journal of Manufacturing Systems (SME), 25(4), pp. 251268, 2006

Yoon, H. S.; Lee, K. T. \& Ahn S. H. (2013). Measurement of roof deformation caused by vehicle rollover, International Journal of Automotive Technology, 14(4), pp. 667-674, Aug 2013 